\title{
X-ray variability of GRS $1915+105$ during the low-hard state observed with the Indian X-ray astronomy experiment (IXAE)
}

\author{
B. Paul ${ }^{1}$, P.C. Agrawal ${ }^{1}$, A.R. Rao ${ }^{1}$, M.N. Vahia ${ }^{1}$, J.S. $\operatorname{Yadav}^{1}$, T.M.K. Marar ${ }^{2}$, S. Seetha ${ }^{2}$, and \\ K. Kasturirangan ${ }^{2}$ \\ 1 Tata Institute of Fundamental Research, Homi Bhabha Road, Mumbai 400 005, India \\ ${ }^{2}$ ISRO Satellite Centre, Airport Road, Vimanpura P.O. Bangalore 560 017, India
}

Received June 9, accepted June 20, 1997

\begin{abstract}
The galactic superluminal transient X-ray source GRS $1915+105$ was observed with the pointed proportional counters (PPCs) onboard the Indian satellite IRS-P3 during 1996 July 23-27. We report here details of the behavior of this source during the relatively quiet and low luminosity state. Large intensity variations by a factor of 2 to 3, generally seen in black-hole candidates, are observed at a time scale of $100 \mathrm{~ms}$ to few seconds. No significant variation is detected over larger time scale of minute or more. The intensity variations are described as sum of shots in the light curve, and the number distribution of the shots are found to be exponential function of the fluence and duration of the shots. The cross correlation spectrum between $6-18 \mathrm{keV}$ and $2-6 \mathrm{keV}$ X-rays is found to have asymmetry signifying a delay of the hard $\mathrm{X}$-rays by about 0.2 to $0.4 \mathrm{~s}$. This supports the idea of hard X-rays being generated by Compton up-scattering from high energy clouds near the source of soft X-rays. Very strong and narrow quasi periodic oscillations in the frequency range 0.62 to $0.82 \mathrm{~Hz}$ are observed. We discuss about a model which explains a gradual change in the QPO frequencies with corresponding changes in the mass accretion rate of the disk.
\end{abstract}

Key words: accretion, accretion disks - black hole physics - X-rays: stars — stars: individual: GRS $1915+105$

\section{Introduction}

The X-ray transient source GRS 1915+105 was discovered in 1992 in hard X-rays using the WATCH all sky X-ray monitor onboard the GRANAT satellite (Castro-Tirado

Send offprint requests to: B. Paul, bpaul@tifrvax.tifr.res.in et al. 1994). Large intensity variations in the source over time scales of a few hours to a few days were detected. During two years of hard X-ray observations by WATCH, two powerful bursts were discovered during which the source luminosity was as high as $10^{39} \mathrm{erg} \mathrm{s}^{-1}$. The hard X-ray spectrum was found to fit well with a power law spectrum with a photon index $(\alpha)$ of -2.5 (Sazonov et al. 1994).

A probable optical counterpart of the source has been observed only in the $I$ band at 23.4 magnitude (Boeer et al. 1996). The optical faintness is probably owing to a low mass companion and very high extinction at a distance of $12.5 \mathrm{kpc} . \mathrm{H}$ and $\mathrm{He}$ emission lines in the infrared were found to be narrow and no Doppler shift was observed. Absence of high velocity signatures in the line profiles in the $I R$ band suggests that the companion is a low mass star (Castro-Tirado et al. 1996). However, high resolution spectral observations in the near-infrared revealed similarity with spectra of very high mass stars of Oe or Be spectral type (Mirabel et al. 1997).

Superluminal motion of two symmetric radio emitting jets of GRS 1915+105 was discovered by Mirabel \& Rodriguez (Mirabel \& Rodriguez 1994) which prompted the object being termed as a micro-quasar. Correlated enhanced radio and hard X-ray emissions were discovered from the source in a long time monitoring during 1994 September to 1996 March (Foster et al. 1996). In a previous outburst during 1993 December to 1994 April decreases or dips in the hard X-ray flux were observed during the radio flares. This observation suggested an interaction between the hard $\mathrm{X}$-ray emission and the jet production. Redirection of the accreted material onto jets can cause the observed X-ray and radio intensity fluctuations (Harmon et al. 1997). Radio flares are thought to be synchrotron emission of outgoing plasmoids from the central object. Infrared jets at the same position angle as that of the radio jets 
were discovered by Sams et al. (1996). The $I-R$ emission from this source is described to be free-free emission of a wind flowing out of the disk. Correlation between the length of a jet, its brightness temperature and central source luminosity was observed which is similar to that seen in AGNs and quasars. This opened up the possibility of understanding the processes in the centers of the AGNs by studying GRS $1915+105$ for which evolution time scale of the jets is a much shorter one.

The X-ray luminosity increased to a very high level in 1996 and the source was observed on several occasions by the pointed proportional counters (PPCs) onboard the Indian satellite IRS-P3 (Agrawal et al. 1996a) and also by PCA and ASM detectors on board the RXTE (Bradt 1996). From 1996 February to March 28 the intensity varied erratically around a mean intensity level of about 1 Crab and this state is termed as "chaotic state". During March 28 to May 17 the source was in a very high intensity state in a declining phase but the intensity variations were relatively less and this is named "bright state" of the source. During May 17 to July 16 very large intensity flares were observed. In this period the X-ray intensity is found to increase by a factor as large as 6 with time scale of a few days. GRS $1915+105$ was found in the same state again during August 15 to December 30, and this state is called "flaring state". In between these two flaring states i.e., during July 16 to August 15 and after the end of the second flaring state until the present time ASM observations indicate a stable state of the source with luminosity about $500 \mathrm{mCrab}$ in the $1.5-12 \mathrm{keV}$ observation range of ASM. The relatively quiet state of the source is termed "low-hard" state because the spectrum was harder during this low state which is common in black hole candidates.

Strong, narrow, quasi periodic oscillations of varying frequency were discovered in GRS $1915+105$ with the PPC observations (Agrawal et al. 1996b; Paul et al. 1997). Intensity dependent narrow QPOs were detected with the PCA. Strong harmonics were also seen at lower frequencies which vanished with increase in intensity and QPO frequency (Chen et al. 1997). The timing behavior, is on some occasions similar to that of the black-hole candidates GS 1124-68 and GX 339-4 (Belloni et al. 1997). The $\mathrm{X}$-ray intensity was found to vary on a variety of time scales ranging from sub-seconds to days during the flaring state, and the spectrum also changed during the brightness variations (Greiner et al. 1996). In the color intensity diagram two separate branches are clearly observed. According to the intensity states, timing behavior and spectral informations, the source has four different states none of which is typical of a black hole state (Morgan et al. 1997).

During our observations in the $2-18 \mathrm{keV}$ energy band spanning 5 days the source was in the low-hard state. The power density spectrum (PDS) of GRS 1915+105 during the low-hard state has a broken power law shape which is almost flat below $1 \mathrm{~Hz}$ and decreases sharply above the QPO frequency (Paul et al. 1997).

\section{Instrument and observations}

The Indian X-ray Astronomy Experiment (IXAE) consists of three pointed proportional counters (PPCs) and one X-ray sky monitor (XSM) (see Agrawal et al. 1997 for more details). The Indian satellite IRS-P3 carrying these instruments along with two remote sensing payloads, was launched with the Polar Satellite Launch Vehicle (PSLV) from Shriharikota (India), on 1996, March 21. The satellite orbits at an altitude of $830 \mathrm{~km}$ with an inclination of $98^{\circ}$ with the equator. Observations of X-ray sources are carried out only in those parts of the orbits which do not go through the South Atlantic Anomaly (SAA) region. The satellite orbit being nearly polar the charged particle background in the detectors increases near the poles and the good observation band is defined to be within the latitude band of $30^{\circ} \mathrm{S}$ to $50^{\circ} \mathrm{N}$. The operating region is chosen to be in this latitude range where the detector background is found to remain constant.

The PPCs have an energy range of $2-18 \mathrm{keV}$ with $60 \%$ efficiency at $6 \mathrm{keV}$. The typical energy resolution of a PPC averaged over the entire detector is $22 \%$ at $6 \mathrm{keV}$. Total photon collecting area of the three detectors is $1200 \mathrm{~cm}^{2}$. The detectors gain can be controlled by changing the high voltage supply in discrete steps and hence the effective energy range can be kept within the desired value. The X-rays detected in each PPC are analyzed in processing electronics units (one for each detector). The processed data, which contain the pulse height histograms and the count rate profiles, are stored in the onboard memories. The timing resolution is different for different modes of observations which is driven by scientific need for the source being observed. The spectral response and detection efficiency of the PPCs has been calibrated by observing the bright X-ray source Crab nebula.

The galactic superluminal X-ray source GRS 1915+105 was observed during 1996 July, 23-27 with the PPCs for a total duration of $8850 \mathrm{~s}$. Observations were limited to the satellite position between the latitude $30^{\circ} \mathrm{S}$ and $50^{\circ} \mathrm{N}$ to avoid high background regions induced by the charged particles. All but one observations were done in a mode with timing resolution of $100 \mathrm{~ms}$, and 64 channel energy spectrum of the source was stored every $10 \mathrm{~s}$. Observation stretches were at most about $20 \mathrm{~min}$ long and in some cases of smaller duration.

\section{Analysis and results}

Background observations were made during August 1-3 in a nearby region of the sky which is devoid of any known $\mathrm{X}$-ray source. When data in the good latitude region of $30^{\circ} \mathrm{S}$ to $50^{\circ} \mathrm{N}$ are taken, the background count rate fits well with a constant value with reduced $\chi^{2} \sim 1.4$. 


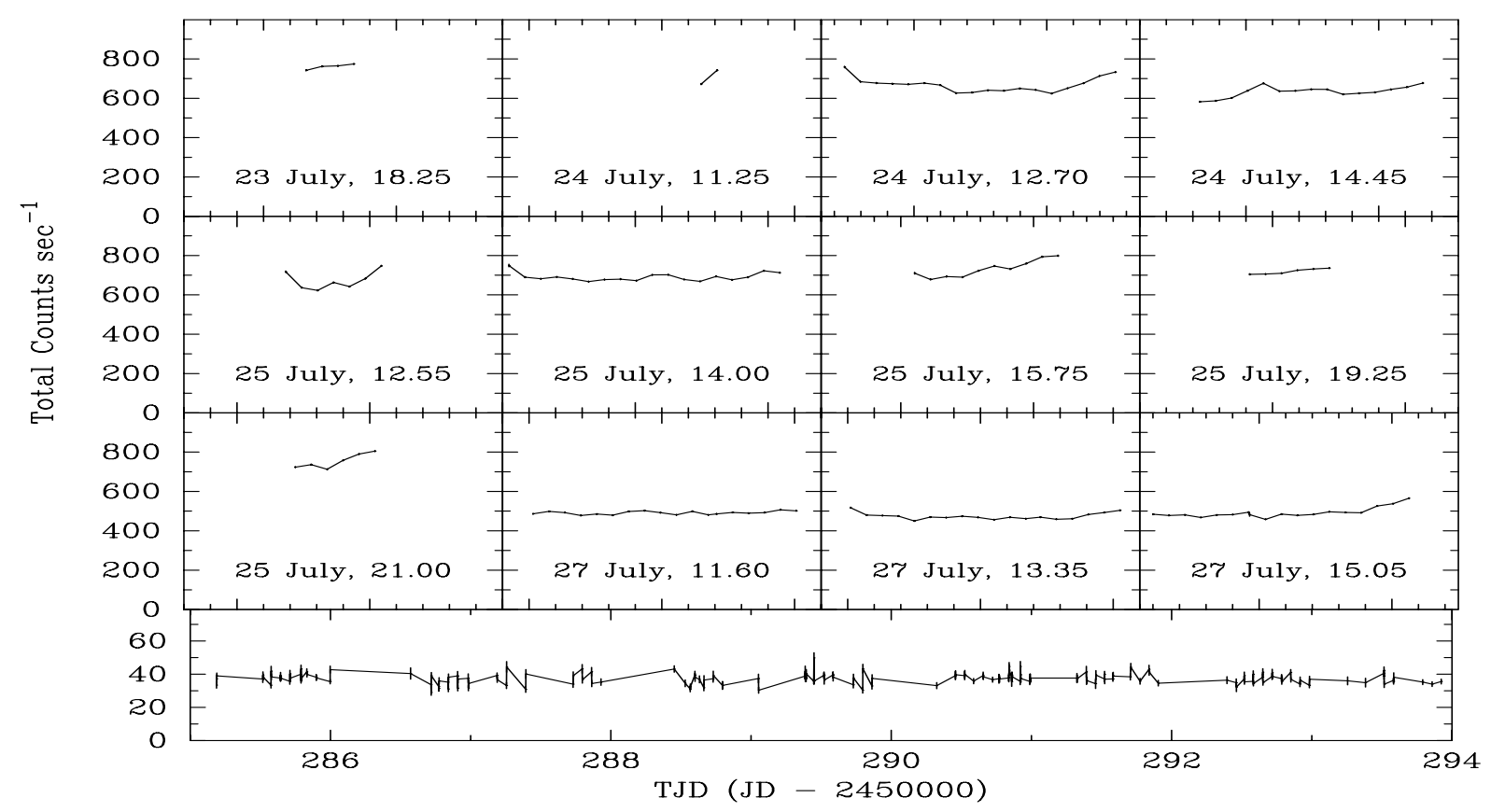

Fig. 1. The light curve of GRS $1915+105$ observed with the PPCs. Date and time of observations are shown in the individual sections. Each section is for 20 minutes duration and data points of bin size of 1 minute are plotted. The bottom panel shows the ASM light curve during July 20-29, each data point is about 90 seconds of observations with about $5-10$ observations every day

Background subtracted light curve is generated for each of the observation slots and the total light curve is shown in Fig. 1. Individual light curve of each observation with integration time of $1 \mathrm{~min}$ are plotted in Fig. 1. The individual observation stretches are 2 to $19 \mathrm{~min}$ long. The date and time of the observations are shown in the panels. The bottom panel shows the ASM light curve of GRS $1915+105$ in $1.5-12 \mathrm{keV}$ range during 1996 July 20-29. Each data point is a result of about 90 seconds observation of ASM with 5-10 observations every day. The ASM light curve of the source, as shown in the lower panel of Fig. 1, is also featureless during the days of our observations. The intensity decrease as observed on July 27 is not evident in ASM data, but the PPC and ASM observations are not exactly simultaneous. Day to day variability in the source intensity is within $10 \%$ of the average value except for the final day of observation. The rms variability in the 1 minute light curve of individual observation slots is only about $1.6 \%$, a part of which is also due to the nonstatistical variation in the background. This small variation can be compared to the $0.5 \% \mathrm{rms}$ variation estimated for a constant intensity light curve of the same intensity with only Poissonian variations.

The hardness ratio, defined as the ratio of counts rate in the $5-12 \mathrm{keV}$ band and $3-5 \mathrm{keV}$ band is found to decrease gradually with few days time scale in the ASM observations during the soft-hard state. We observe no noticeable change in the hardness ratio during individual

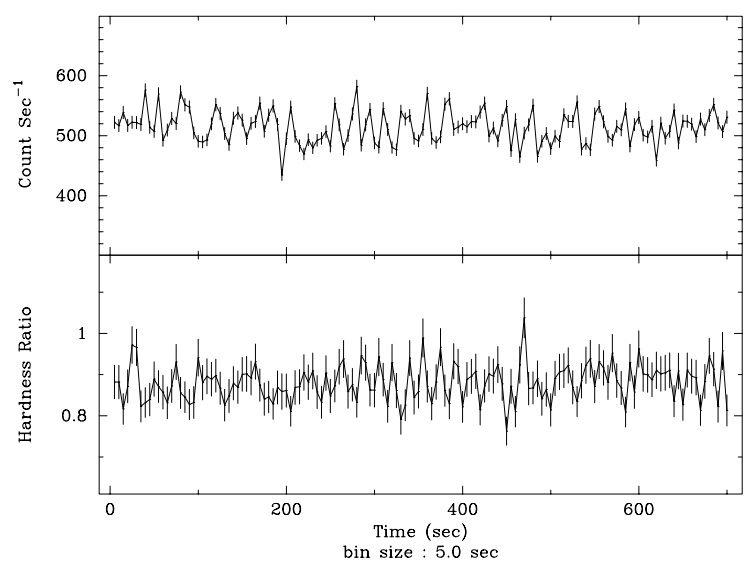

Fig. 2. The intensity and hardness ratio are plotted for one of the PPC observation slots. Data bin size is chosen to be 5 seconds to reduce the statistical errors

observation slots. In Fig. 2 we have shown a section of the light curve with the hardness ratio plotted with it. The hardness ratio is defined as the number of counts in $6-18 \mathrm{keV}$ divided by those in $2-6 \mathrm{keV}$. A bin size of 5 seconds has been used to compute the hardness ratio to reduce the error bars.

A search was made to find intensity variations in the source largely exceeding the photon counting statistics. Each individual time bin was inspected with respect to a 

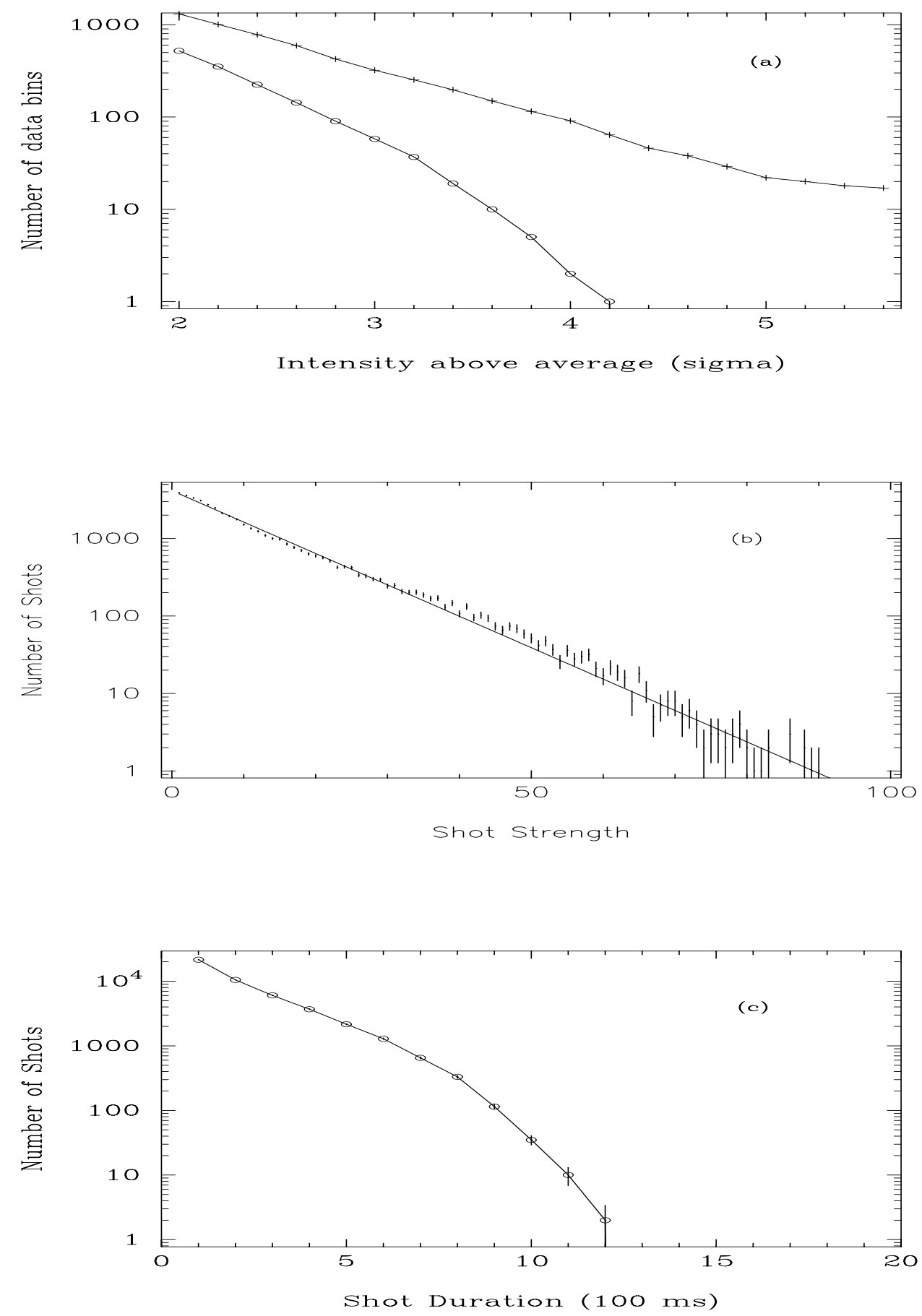

Fig. 3. A statistical representation of the intensity variations in GRS $1915+105$ observed with the PPCs. The data sets of the three detectors are treated as independent observations and the results are added. a). The number of $100 \mathrm{~ms}$ data bins exceeding a running average of 21 data points is plotted as a function of the excess expressed in $\sigma$. The lower curve is the expected distribution calculated with a synthetic light curve with the same running average as in the observed light curve, $\mathbf{b}$ ). the number distribution of the shots as a function of number of photons in the shots and $\mathbf{c}$ ). the number distribution of shots as a function of shot duration 


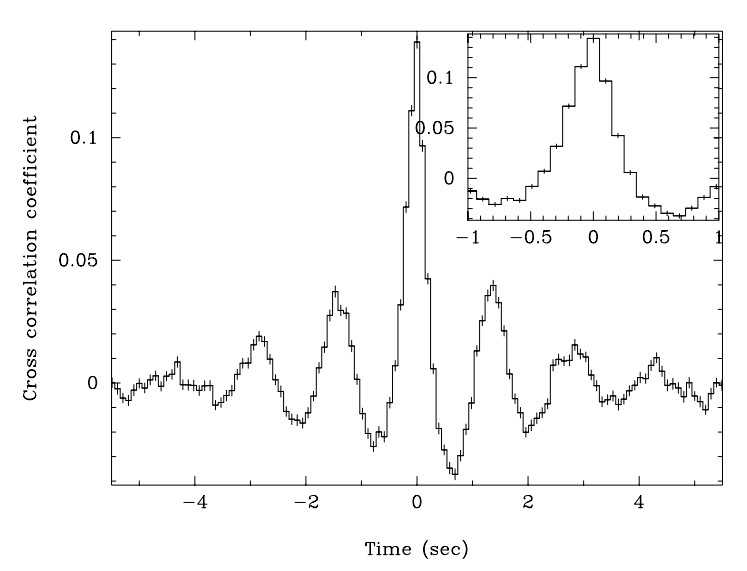

Fig. 4. The cross correlation function for different delays between the soft X-rays $(2-6 \mathrm{keV})$ and hard X-rays $(6-18 \mathrm{keV})$ is plotted here. The oscillations in the plot is due to the QPOs at a period of 1.4 seconds. In the inset is shown the cross correlation function near zero, asymmetry on the two sides of zero is visible

running average in the light curve around that bin and intensity variations above the average were classified in terms of its strength. In Fig. 3a we have shown the number distribution of data bins exceeding the average, as a function of the excess. The number of data points where large intensity enhancement is detected is much more than that expected in an otherwise constant intensity light curve with Poissonian statistics. This difference is more pronounced for the larger intensity enhancements. We have earlier reported large intensity variations over time scale of $100 \mathrm{~ms}$ to few sec in our observation of GRS $1915+105$ (Paul et al. 1997). However there is no intensity variation at a longer time scale of a minute or more as can be seen in Fig. 1 where one minute count rate is plotted.

Time variability in the X-ray intensity of black hole sources has been proposed to be the result of randomly occurring shots with exponential rise and/or decay (Terrell 1972). A Large number of shots in the Cyg X -1 light curve were added and the resultant profile was found to have nearly symmetric rise and decay (Negoro et al. 1994). To quantify the variations in the intensity as sum of shots in the light curve of GRS $1915+105$, we have identified shots and classified them in terms of the number of photons in them. Every data bin of the $100 \mathrm{~ms}$ light curve is compared to a running average around it, and successive data bins, when found to be above the average, a shot is presumed to have occurred. The total excess counts in the individual shots above the average are calculated and a number distribution of that is shown in Fig. $3 \mathrm{~b}$. We find that the distribution fits very well with an exponential function $(f(S)=N \exp -S / C$; with $N=4140$ and $C=10.7 ; \mathrm{S}$ is the strength of the shot in photon counts). The durations of the shots are shown in Fig. 3c which also has an exponential form with increasing slope above 0.7 seconds. The shape of the curve in Fig. 3c can also be explained as an exponential distribution of shot duration with a hump around $0.7 \mathrm{~s}$, which is the width of the pulse profile at the quasi-periodic oscillation period of 1.4 second.

To measure any delay between the hard and soft $\mathrm{X}$-rays, cross correlations (correlation coefficients with different delays) were calculated. All observation slots were divided into smaller segments of 64 data points of $100 \mathrm{~ms}$ duration. The cross correlation function between the $2-6 \mathrm{keV}$ and $6-18 \mathrm{keV}$ count rate profiles were calculated for all of these small data lengths. The resultant cross correlation functions were added and averaged and are plotted in Fig. 4. The peaks in the cross-correlation plot are due to the strong QPOs at a frequency of $0.7 \mathrm{~Hz}$. The region near 0 is plotted in the inset and the asymmetry around 0 delay is clearly visible. One possible explanation for this asymmetry in the cross correlation function is a time lag between the soft and hard X-ray oscillations. The difference between the right and left hand side of the cross correlation function is maximum at around $0.2-0.4 \mathrm{~s}$ indicating a delay of 0.2 to $0.4 \mathrm{~s}$ for the hard $\mathrm{X}$-rays compared to the soft X-rays in our observations. Similar asymmetry is observed in all the observations and in all the three detectors.

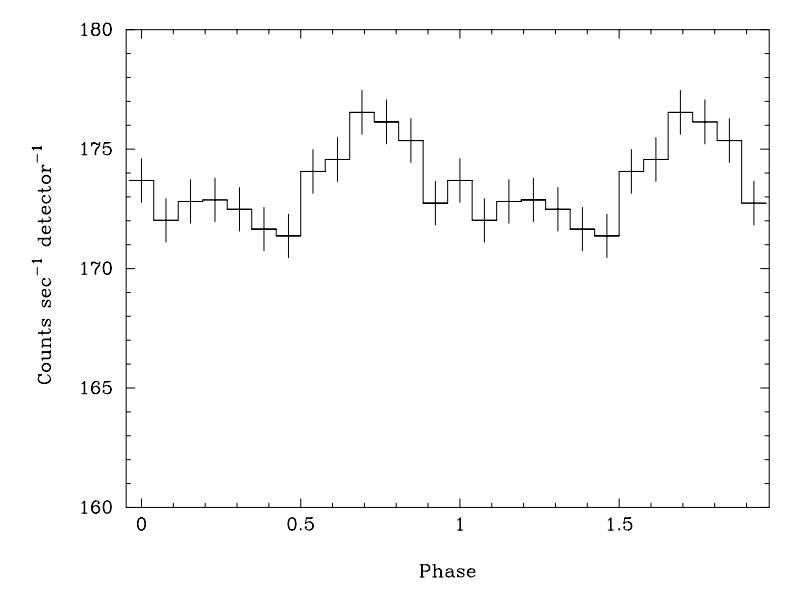

Fig. 5. The pulse profile of GRS $1915+105$ folded with the mean QPO period

We have discovered quasi-periodic oscillations in GRS $1915+105$ with frequency varying between 0.62 to $0.82 \mathrm{~Hz}$ (Agrawal et al. 1996b; Paul et al. 1997). GRS $1915+105$ is the sixth black hole candidate after GX $339-4$, Cyg X - 1, LMC X - 1, GS 1124-68 and GRO J0422+32, in which QPOs have been observed. The power density spectrum obtained from the PPC observations shows that the QPOs are very narrow $(<0.2 \mathrm{~Hz})$ and strong ( $\mathrm{rms} 9 \%)$. The PDS is flat for frequencies less than the $\mathrm{QPO}$ frequency and at frequencies 
above this it follows a power law of index -1.5 . There is no marked difference between the power spectrum of the low and high energy X-rays. The PDS of GRS 1915+105 as observed in its low state resembles that of the other black hole candidates in their very high state. This type of band limited noise is characteristic of black hole sources in their very high state (Miyamoto et al. 1992; van der Klis 1995). Black hole sources have strong very low frequency intensity variations when in low-hard state and in high state the PDS is flat below a break frequency.

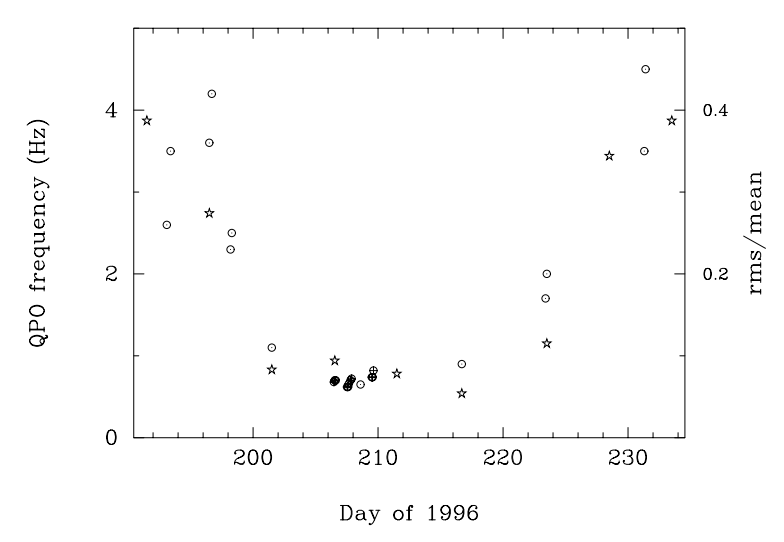

Fig. 6. The QPO frequency (left scale) and rms variation (right scale) history of GRS 1915+105 during the low-hard state. The crossed circles are PPC observations and dotted circles are PCA observations. The stars represent the rms/mean during a 6 days period in the ASM light curve around each point

The light curve was folded at the observed QPO period and the resultant profile is found to be nearly sinusoidal with pulse amplitude of $4 \%$ of the total intensity of the source (Fig. 5). There is no noticeable difference in pulse shape in the two energy ranges, $2-6$ and $6-18 \mathrm{keV}$.

\section{Discussion}

Large intensity variations over very short time scales are present in many black-hole candidates and in some neutron star systems. In the neutron star systems if the magnetic field is weak, the inner radius of the accretion disk can be very close to the neutron star surface resulting in flux variation at the Keplerian frequencies, or at the beat frequencies between the Keplerian frequency and spin of the neutron star. In the case of black-hole sources, the disk can extend upto the lowest stable orbit (3 times the Schwartzchild radius) for a non-rotating black hole, or even closer to the compact object for a maximally rotating compact object. The fast variations observed in GRS $1915+105$ indicate size of the emission region $\lesssim 0.1$ light second, large for the inner disk of a few stellar mass black-hole.

The hard X-rays are found to be delayed compared to the soft X-rays indicating that the soft and hard $\mathrm{X}$-ray emitting regions are not the same and the variations in luminosity of the two regions are not simultaneous, as it is expected to be for physically separated regions. The hard X-ray emissions from black hole candidates are usually thought to be reprocessed emission of underlying soft photons from an inner disk. In such cases multiple inverse Compton scattering by the energetic electrons in the corona, which is responsible for the increase in energy of the photons, may introduce some delay between the soft and hard X-ray spectrum. Our observation of hard $\mathrm{X}$-ray delay in the source, which is overlying on the QPOs as shown in Fig. 4, indicates that the soft X-rays (most of them of energy $<6 \mathrm{keV}$ ) from the inner disk are upscattered by a plasma of size of a fraction of a light-second.

Quasi-periodic oscillations of many different frequencies are observed in GRS 1915+105 (Morgan et al. 1997). During the bright state, low frequency QPOs were observed with harmonics. QPOs of two or more different frequencies were also observed. The QPO frequency in the flaring state varied erratically between $0.0016 \mathrm{~Hz}$ and $0.16 \mathrm{~Hz}$ with a higher frequency QPO of $7.6 \mathrm{~Hz}$ observed on May 14. In the two flaring states the QPO frequency is relatively high, $1.5 \mathrm{~Hz}$ to $8 \mathrm{~Hz}$ varying erratically with a low frequency QPO of $0.003 \mathrm{~Hz}$ observed on June 16 . The $67 \mathrm{~Hz}$ QPOs are observed during the bright and flaring state. The QPOs frequency variations during the low-hard state, seems to have some definite pattern in it. The QPO frequency was $2.3 \mathrm{~Hz}$ at the beginning of the low-hard state, decreased slowly to a lowest frequency of $0.62 \mathrm{~Hz}$ on July 25 , and again increased slowly to $2.0 \mathrm{~Hz}$ towards the end of the low-hard state. The QPO frequency history of GRS $1915+105$ during its low state inferred from published data is shown in Fig. 6. The rms/mean of 6 days observations of ASM around each data point is plotted along with the QPO frequency. Crossed circles are PPC observations, the dotted circles represent PCA observations (Morgan et al. 1997) and the stars are the rms/mean obtained from ASM archival data. We notice that the QPO frequency decreased to a minimum during our observations and then increased again. Erratic changes in the QPO frequency between segments of observations on the same day are present. An overall trend of a smooth decrease followed by increase in the QPO frequency is clearly observed. The rms deviation of the X-ray intensity shows dramatic change when the source makes transition from flaring to low state and again back to the flaring state. The QPO frequency appears to be very strongly related to the rms/mean intensity of the source. The hardness ratio between the $5-15 \mathrm{keV}$ flux and $3-5 \mathrm{keV}$ flux increased during the low-hard state and decreased again as the source went to the flaring state again. 
The quasi-periodic oscillations detected during our observations are stable, narrow and strong. It may represent the Keplerian motion of blobs of hot material in the disk. Stability of the QPO frequency in the range 0.62 to $0.82 \mathrm{~Hz}$ during 5 days of observations, during which the overall intensity of the source is also nearly constant, indicates that the QPO production region is narrow in the radial direction and is stable when the mass accretion rate is stable. But the force behind confinement of the hot spots in the radial directions which can produce narrow QPOs, is not understood.

In a model of disk accretion around black hole sources, in which the disk passes through a standing shock close to the centrifugal barrier, a QPO generation mechanism has been developed (Molteni et al. 1996). Oscillations around the mean shock location can give rise to intensity modulations similar to the quasi periodic oscillations observed in the black hole candidates like GX 339 - 4, GS 1124-68 etc. The oscillations of the shock surface arise as a balancing act between the infall of the converging disk material and the outflow of the hot shockfront. If the cooling time scale of the expanding postshock halo and the infall time scale of the disk are nearly the same, a modulation of upto $10-15 \%$ of the intensity can be produced. In this model the QPO frequency is predicted to increase with mass accretion rate. Sustained quasi-periodic oscillations are possible if the dominant cooling process is bremsstrahlung. Chakrabarti \& Titarchuk (1995) have shown that for sources emitting near their Eddington luminosity the post shock region emits mainly by bremsstrahlung. The smooth QPO frequency variations during the low-hard state can be attributed to a similar increase in the mass accretion rate. This is in agreement with the QPO production process mentioned above. In this model the spectrum becomes harder at lower luminosity. This fact also has been observed in the ASM data showing a harder spectrum during the low state (Bradt 1996). For a $33 M_{\odot}$ black hole (for a stable $67 \mathrm{~Hz}$ QPO observed with PCA to represent the Keplerian rotation of the innermost stable disk around a non-rotating black hole) and $0.7 \mathrm{~Hz}$ QPO, the average shock front is at a radius of around $100 r_{\mathrm{g}}$.

\section{Conclusion}

In our 4 days of observations of the galactic superluminal source GRS $1915+105$ in the energy range $2-18 \mathrm{keV}$, we have observed intensity variations over time scale of $100 \mathrm{~ms}$ to few seconds. The hard X-rays are found to have time lag compared to the soft X-rays supporting the Compton up-scattering models of hard X-ray production in black-hole candidates. Strong QPOs in a narrow range of $0.62 \mathrm{~Hz}$ to $0.82 \mathrm{~Hz}$ are from a narrow region in the disk which is stable during the quiescent state of this source. We show that the observed QPO frequency variations can be explained if the QPOs are generated by the oscillations of the shock front in the accretion disk.
Acknowledgements. We gratefully acknowledge the support given by Shri K. Thyagrajan, the Project Director of IRSP3 satellite, Shri R.N. Tyagi, Manager IRS programme, Shri R. Aravamudan, Director, ISAC and other technical and engineering staff of ISAC in making the IXAE project a success. We particularly thank M.R. Shah, J.P. Malkar, K. Mukerjee, D.K. Dedhia and P. Shah of our group and N. Upadhyaya, M.R. Sharma, N.S. Murthy, C.N. Umapathy and L. Abraham of Technical Physics Devision, ISAC, and other engineering, scientific and technical staff of other divisions of ISAC who were involved in the IRS-P3 project and ISRO tracking facility, for their efforts in making this experiment successful.

\section{References}

Agrawal P.C., Paul B., Rao A.R., et al., 1996a, J. Korean Astron. Soc. 29, S429

Agrawal P.C., Paul B., Rao A.R., et al., 1996b, IAUC No. 6488 Agrawal P.C., Paul B., Rao A.R., et al., 1997, Current Sci. (in preparation)

Belloni T., van der Klis M., Lewin W.H.G., et al., 1997 (to appear in A\&A)

Bradt H., 1996, Proceedings of 5th International Workshop on Data Analysis in Astronomy, CCSEM Center, Erice, Italy, Scarsi L. and Maccarone C. (eds.). World Scientific Publ. Co.

Boeer M., Greiner J., Motch C., 1996, A\&A 305, 835

Castro-Tirado A.J., Brandt S., Lund N., et al., 1994, ApJS 92, 469

Castro-Tirado A.J., Geballe T.R., Lund N., 1996, ApJ 461, L99

Chakrabarti S., Titarchuk L.G., 1995, ApJ 455, 623

Chen X., Swank J.H., Taam R.E., 1997, ApJ 477, L41

Finoguenov A., Churazov E., Gilfanov M., et al., 1994, ApJ 424,940

Foster R.S., Waltman E.B., Tavani M., et al., 1996, ApJ 467, L81

Greiner J., Morgan E.H., Remillard R.A., 1996, ApJ 473, L107

Harmon B.A., Deal K.J., Paciesas W.S., et al., 1997 ApJ 477, L85

Mirabel I.F., Rodriguez L.F., 1994, Nat 371, 46

Mirabel I.F., Bandyopadhyay R., Charles P.A., Shahbaz T., Rodrigues L.F., 1997, ApJ 477, L45

Miyamoto S., Kitamoto S., IGA S., Negoro H., Terada K., 1992, ApJ 391, L21

Molteni D., Sponholz H., Chakrabarti S.K., 1996, ApJ 457, 805

Morgan E.H., Remillard R.A., Greiner J., 1997 (to appear in ApJ)

Negoro H., Miyamoto S., Kitamoto S., 1994, ApJ 423, L127

Paul B., Agrawal P.C, Rao A.R., et al., 1997, A\&A 320, L37

Sams B.J., Eckart A., Sunyaev R., 1996, Nat 382, 47

Sazonov S. Yu., Syunyaev R.A., Lapshov I. Yu., et al., 1994, Astron Lett. 20, 787

Terrell N.J., 1972, ApJ 174, L35

van der Klis M., 1995, in X-ray Binaries, Lewin. W.H.G., jan van Paradijs \& van den Heuvel (eds.). Cambridge University Press, pp. 252-300 